\title{
Fast Tracking in Off Pump CABG with Supraglottic Airway and TIVA
}

\author{
Kalpana Shah ${ }^{1}$ \\ ${ }^{1}$ Cardiac Anaesthesiologist, Breach Candy Hospital, Mumbai, India \\ J Card Crit Care:2021;5:45-48
}

\begin{abstract}
Address for correspondence Kalpana Shah, MBBS, DA, DNB, Cardiac Anaesthesiologist, Breach Candy Hospital, Mumbai, India (e-mail: kalps99ana@gmail.com).
\end{abstract}

\begin{abstract}
Keywords

- fast tracking

- off pump CABG

- supraglottic airway

- TIVA

The standard goals of anesthetic management for an off pump coronary artery bypass (OPCAB) surgery are that it should be safe, provide cardiac and other organ protection and stability, preserve neurocognitive integrity, maintain hemodynamics, allow early emergence and ambulation, and offer pain relief in the postoperative period. The cardiac surgical team should collaborate and plan the best preoperative strategy, so as to provide optimal care and a rapid and expeditious recovery of these patients. Scientific evidence and practical experience make total intravenous anesthesia (TIVA) the recommended choice for cardiac surgery. Use of endotracheal tube (ETT) is associated with certain drawbacks which can be overcome with judicious use of ProSeal laryngeal mask airway (PLMA) for cardiac surgeries too. The core principles of ultrafast track anesthetic (UFTA) technique are choice and titration of short-acting anesthetic drugs, postoperative normothermia, multimodal analgesia, early extubation, ambulation, and discharge. Fast tracking with use of TIVA and PLMA in patients undergoing OPCAB at our center offers certain advantages over other techniques in terms of reduced airway and lung trauma, improved cardiac output and renal perfusion with spontaneous respiration, decreased stress and discomfort of ETT suctioning and weaning from ventilation, substantially reduced requirement of dosages of analgesics, anesthetics and opioids, resulting in better patient hemodynamics, alert and pain-free patient that are more amenable and cooperative for early feeds, postoperative chest physiotherapy and lung recruitment strategies, and early ambulation and discharge.
\end{abstract}

\section{Introduction}

"Change is the only constant in life."-Heraclitus

Over the last two decades, the shape of anesthesia for cardiac surgery has undergone significant changes from opioid-dominated technique and high-thoracic epidural anesthesia (HTEA) to now a more balanced and multimodal anesthesia, offering fast tracking. The evolution of techniques and knowledge of beating heart surgery has led to innovations, in order to promote patient safety and ensure high standards of care. The standard goals of anesthetic management for an off pump coronary artery bypass (OPCAB) surgery are

published online March 27, 2021
DOI https://doi.org/

$10.1055 / \mathrm{s}-0041-1724147$ ISSN 2457-0206. that it should be safe, provide cardiac and other organ protection and stability, preserve neurocognitive integrity, maintain hemodynamics, allow early emergence and ambulation, and offer pain relief in the postoperative period. The cardiac surgical team should collaborate and plan the best preoperative strategy, so as to provide optimal care and a rapid and expeditious recovery of these patients. ${ }^{1}$

\section{Ideal Anesthetic Agent}

The search for an ideal anesthetic agent is never ending.

The ideal anesthetic agent should encompass properties such as rapid onset and offset, rapid emergence and recovery

(c) 2021. Official Publication of The Simulation Society (TSS), accredited by International Society of Cardiovascular Ultrasound (ISCU).

This is an open access article published by Thieme under the terms of the Creative Commons Attribution-NonDerivative-NonCommercial-License, permitting copying and reproduction so long as the original work is given appropriate credit. Contents may not be used for commercial purposes, or adapted, remixed, transformed or built upon. (https://creativecommons.org/licenses/by-nc-nd/4.0/)

Thieme Medical and Scientific Publishers Pvt. Ltd., A-12, 2nd Floor, Sector 2, Noida-201301 UP, India 
to baseline, be easily titrated, analgesia at subanesthetic concentrations, cause minimal or no cardiovascular and respiratory depression, have organ independent metabolism with no active metabolites, have no toxic effects on other organs, have no interaction with neuromuscular blocking drugs, not cause hypersensitivity reaction or histamine release, have antiemetic, antioxidant, anti-inflammatory effects, have long shelf life, and be atmosphere friendly (green). ${ }^{2}$

\section{Current Scenario}

Modern anesthesia is still mostly administered by the inhalational route but there is increasing concern over their potential for pollution and other adverse effects. Exposure to halogenated hydrocarbons may cause reduction in antioxidant activity in plasma and erythrocytes, inhibition of neutrophil apoptosis, depression of central neurorespiratory activity, increased DNA breaks, and effects on cerebral blood circulation and altered renal function.

Research as of today advocates use of volatile agents as the preferred choice in cardiac anesthesia for myocardial protection; however, this science has not been convincingly translated into clinical practice. ${ }^{1}$ Myriad anesthetic techniques have been studied for patients undergoing OPCAB, ranging from HTEA with endotracheal tube (ETT) to only HTEA in conscious patients. ${ }^{3}$

\section{Anesthetic Approaches in OPCAB}

The anesthetic approaches that have been developed and utilized over period of time for patients undergoing OPCAB are general anesthesia (GA) with opioids and inhalation anesthesia; GA combined with controlled ventilation and HTEA; GA combined with intrathecal opioids; awake regional anesthesia with spontaneous ventilation using HTEA alone; total intravenous anesthesia (TIVA) with ETT; and TIVA with laryngeal mask airway (LMA). ${ }^{3-7}$

Scientific evidence and practical experience make TIVA the recommended choice for cardiac surgery ( - Fig. 1). ${ }^{8}$

As a standard practice, most patients are intubated using standard ETT for upper airway management, however, use of ETT is associated with certain challenges for an anesthetist such as increased sympathetic response, resulting in hemodynamic aberrations, which leads to requirement of higher

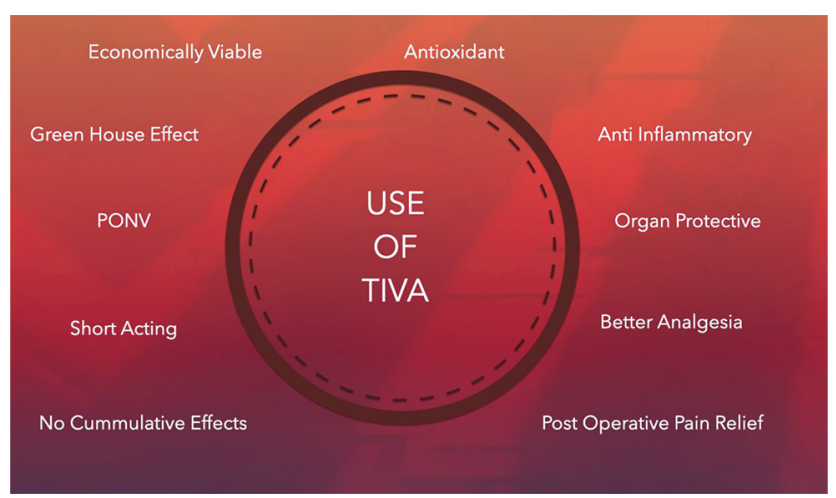

Fig. 1 Benefits of using total intravenous anesthesia (TIVA) in patients. doses of drugs such as opioids, vasodilators, $\beta$-blockers or inotropes, in order to maintain acceptable hemodynamics parameters; thus, there associated side effects, use of drugs for maintaining tube compliance, adverse effects such as laryngospasm, bronchospasm and vocal cord related issues, and issues related to extubation.

Thus, it is the need of hour to develop safe anesthetic techniques, whereby use of ETT and its associated drawbacks can be avoided.

\section{Laryngeal Mask Airway (LMA)}

The drawbacks associated with the use of ETT led the team at our center to explore for a safe alternative to ETT. Since LMA are used rampantly in noncardiac surgeries, the team started evaluating LMA as an alternative to ETT for cardiac surgeries too.

The basis for selecting LMA was that it is a safe alternative for airway management, can be considered for postoperative ventilation, and better hemodynamic control; thus, there is decreased requirement of dosages of opioids, analgesics, anesthetics and inotropic agents, negligible to no use of $\beta$ blockers intraoperatively with use of dexmedetomidine, and no airway-related issues. Therefore, patient compliance is better. $3,4,9$

\section{Ultrafast Track Anesthetic (UFTA) Technique}

Fast tracking in cardiac surgery is an accepted technique due to improvement in resource utilization. Anesthesia management tailored to facilitate fast tracking in cardiac surgery is routinely practiced now. UFTA refers to extubation within 2 hours of the end of surgery. The core principles of UFTA are choice and titration of short-acting anesthetic drugs, postoperative normothermia, multimodal analgesia, and early extubation, ambulation and discharge. It is gaining importance because of rapid mobilization, less morbidity, early discharge, better patient comfort, and economic concerns. Ultrafast tracking algorithm in cardiac anesthesia has significantly fastened the postoperative recovery..$^{10,11}$

\section{Benefits of Fast Tracking with Use of TIVA and PLMA}

TIVA with LMA offers certain advantages over other techniques in terms of reduced airway and lung trauma, improved cardiac output and renal perfusion with spontaneous respiration, decreased stress and discomfort of ETT suctioning and weaning from ventilation, substantially reduced requirement of dosages of analgesics, anesthetics and opioids, resulting in better patient hemodynamics, alert and pain-free patients who are more amenable and cooperative for early feeds, postoperative chest physiotherapy and lung recruitment strategies, and early ambulation and discharge.

\section{Fast Tracking with use of TIVA and PLMA at our Center} Patient Selection

To achieve good outcomes with the use of TIVA along with LMA in patients undergoing OPCAB, it is must to set ideal patient selection criteria. At our center, OPCAB under TIVA 
with LMA is contraindicated in patients with gastroesophageal reflux disease, hiatus hernia, full stomach, morbid obesity, cervical spine fracture, glottic or subglottic obstruction, modified Mallampati class (MPC) 2 or 3, history of major gastric surgery, need for transesophageal echo, left ventricular ejection fraction less than $20 \%$, arrhythmias, unstable angina requiring intra-aortic balloon pump (IABP)/high inotropic support, chronic atrial fibrillation, chronic liver/renal disease, those undergoing emergency bypass, and those with crashed angioplasty, apart from the routine contraindications for LMA.

\section{Prehabilitation of Patients}

Prehabilitation is the process of enhancing physiological, nutritional, and psychological resilience to increase patients' functional capacity in advance of a known physiologically stressful event. It aims to empower patients, reduce the risk of postoperative morbidity, improve the rate of postoperative recovery and, ultimately through sustained behavioral change and improved management of comorbidities, improve long-term health outcomes.

At our center, as a part of prehabilitation, the patients were counseled and encouraged for smoking and alcohol cessation and adopting healthy diet. Blood sugar and blood pressure was optimally controlled. Anticoagulants were stopped for the patients. They were given steam inhalation, chest physiotherapy, incentive spirometry, and nebulization (as required).

\section{Perioperative Management}

Equally important to prehabilitation is perioperative care of patients. At our center, the procedures undertaken as a part of perioperative management are as follows:

- Angiotensin-converting enzyme inhibitors (ACEI) and angiotensin II receptor blockers (ARBs) are omitted 24 hours prior to scheduled surgery.

- Antiplatelet drugs like aspirin, clopidogrel, etc., are withheld at least 5 days prior to surgery

- On the night prior to surgery, pantoprazole $40 \mathrm{mg}$ and alprazolam $0.5 \mathrm{mg}$ are given to the patients

- On the day of surgery, in the operating room, monitoring noninvasive and invasive lines are secured under sedation with $0.02 \mathrm{mg} / \mathrm{kg}$ midazolam, $1 \mathrm{~g}$ paracetamol, and $1 \mathrm{~g}$ Mgso4.

- Patients are induced with fentanyl (1-2 $\mu / \mathrm{kg})$ and dexmedetomidine ( 0.5 to $1 \mu / \mathrm{kg}$, can be avoided if patients are well $\beta$ blocked), have low ejection fraction (<35\%), or have arrhythmia, propofol (medium-chain triglyceride/ long-chain triglyceride [MCT/LCT]) (0.5 mg-1 mg/kg) and atracurium $(0.3-0.6 \mathrm{mg} / \mathrm{kg})$.

- Following induction, patients are maintained with infusion of fentanyl (0.5-1 mcg/kg), propofol (0.05-0.1 mg/kg) and dexmedetomidine $(0.5-1 \mathrm{mcg} / \mathrm{kg})$; doses of which are titrated in accordance to hemodynamic response.

- Patients are ventilated with a mixture of air and oxygen.
- Patients are monitored using five-lead ECG, arterial pressure, central venous pressure or pulmonary artery pressure and wedge pressure, pulse oximetry, capnography, and core temperature, which are used routinely for monitoring patients undergoing OPCAB surgery.

- Bispectral index (BIS) is measured to maintain a BIS value of 40 to 50.

- ProSeal LMA (PLMA) is introduced using gum elastic bougie technique and railroading the LMA over it. The positioning is confirmed by visual chest expansion, end-tidal carbon dioxide $\left(\mathrm{Et}^{\mathrm{CO}} \mathrm{O}_{2}\right.$ ), expiratory tidal volume monitoring on ventilator screen and performing blob test (lack of bobbing of gel indicates tip of drain tube is not in direct continuity with upper esophagus, suggesting the mask tip has folded over). ${ }^{12}$ Finally, the Ryles tube (RT) is placed from the double-lumen end of the LMA.

\section{Extubation Criteria}

The patients can be extubated if they are cooperative and alert, have oxygen saturation (SpO2) $\geq 98 \%$, have $\mathrm{FiO} 2<60 \%$ and $\mathrm{Et}_{\mathrm{CO}_{2}}<45 \mathrm{~mm} \mathrm{Hg}$, have stable hemodynamics with minimal inotropes, normal arterial blood gas analysis, and do not have arrhythmias or hypothermia.

\section{Postoperative Pain Management}

For postoperative pain management, patients are administered opioid analgesics (injection fentanyl as infusion at a dose of $0.5-1 \mu \mathrm{g} / \mathrm{kg} / \mathrm{hr}$ ) along with injection ondansetron $8 \mathrm{mg}$ as an infusion (rate of administration is determined by weight of patient) (-Table $\mathbf{1}$ ). IV paracetamol $1 \mathrm{gm}$ (in $100 \mathrm{~mL}$ ) is administered four times a day (QID), while nonsteroidal anti-inflammatory drugs (NSAIDs) like diclofenac are given in patients with normal renal functions. IV tramadol $50 \mathrm{mg}$ is administered as and when required in those with pain despite above medications.

\section{ICU Discharge Criteria's}

Patients are discharged once they are hemodynamically stable, have adequate urine output, have adequate pain control, do not require inotropes or pacemaker, do not have arrhythmias, and have all invasive lines removed once (-Fig. 2).

Table 1 Infusion rate based on weight of patients

\begin{tabular}{|l|l|l|l|}
\hline Weight $(\mathrm{kg})$ & $\begin{array}{l}\text { Infusion rate } \\
(\mathrm{mL} / \mathrm{hr})\end{array}$ & Weight $(\mathrm{kg})$ & $\begin{array}{l}\text { Infusion rate } \\
(\mathrm{mL} / \mathrm{hr})\end{array}$ \\
\hline 35 & 3.5 & 70 & 7 \\
\hline 40 & 4 & 75 & 7.5 \\
\hline 45 & 4.5 & 80 & 8 \\
\hline 50 & 5 & 85 & 8.5 \\
\hline 55 & 5.5 & 90 & 9 \\
\hline 60 & 6 & 95 & 9.5 \\
\hline 65 & 6.5 & 100 & 10 \\
\hline
\end{tabular}




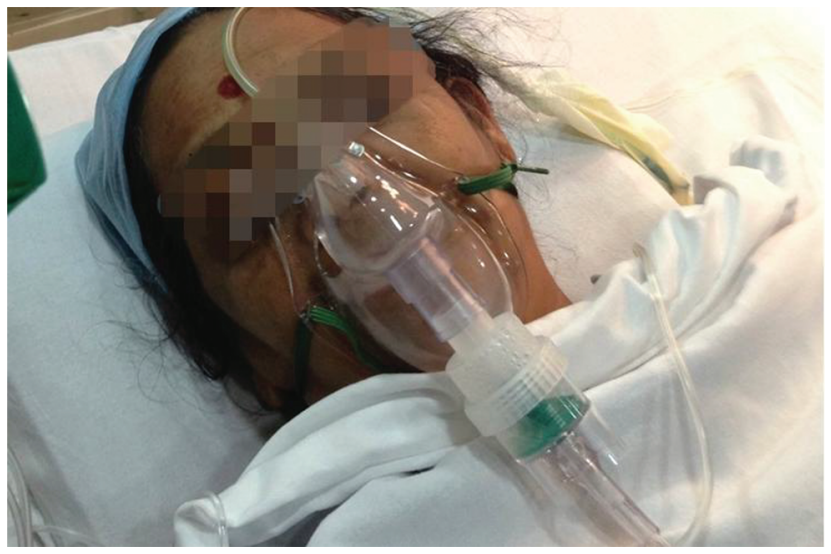

Fig. 2 Patient that underwent successful off pump coronary artery bypass (OPCAB) surgery under total intravenous anesthesia (TIVA) with ProSeal laryngeal mask airway (PLMA).

\section{Conclusion}

Use of TIVA with PLMA in patients undergoing OPCAB is associated with advantages such as lower pain scores, decreased requirement of muscle relaxants, decreased requirement of intraoperative $\beta$ blockade, and decreased use of analgesics/opioids and vasoactive/vasodilator drugs. In the ideal patients, judicious use of this technique will serve the purpose of UFTA.

"A new idea is first condemned as ridiculous and then dismissed as trivial, until finally, it becomes what everybody knows."-William James

\section{Conflicts of Interest}

None declared.

\section{References}

1 Suryaprakash S, Chakravarthy M, Muniraju G, et al. Myocardial protection during off pump coronary artery bypass surgery: a comparison of inhalational anesthesia with sevoflurane or desflurane and total intravenous anesthesia. Ann Card Anaesth 2013;16(1):4-8

2 Min JJ, Kim G, Lee JH, Hong KY, Kim WS, Lee YT. Does the type of anesthetic technique affect in-hospital and one-year outcomes after off-pump coronary arterial bypass surgery? PLoS One 2016;11(4):e0152060

3 Chakravarthy M, Jawali V, Patil TA, et al. Conscious cardiac surgery with cardiopulmonary bypass using thoracic epidural anesthesia without endotracheal general anesthesia. J Cardiothorac Vasc Anesth 2005;19(3):300-305

4 Shah K. ProSeal laryngeal mask airway as an alternative to standard endotracheal tube in securing upper airway in the patients undergoing beating-heart coronary artery bypass grafting. Ann Card Anaesth 2017;20(1):61-66

5 Shah K, Kulshreshtha A. Comparison of rocuronium and vecuronium in patients undergoing elective ultrafast-track off-pump coronary artery bypass surgery. Journal of Cardiac Critical Care TSS. 2020;3(2):068-076

6 Chakravarthy M, Thimmangowda P, Krishnamurthy J, Nadiminti $\mathrm{S}$, Jawali V. Thoracic epidural anesthesia in cardiac surgical patients: a prospective audit of 2,113 cases. J Cardiothorac Vasc Anesth 2005;19(1):44-48

7 Hemmerling TM, Romano G, Terrasini N, Noiseux N. Anesthesia for off-pump coronary artery bypass surgery. Ann Card Anaesth 2013;16(1):28-39

8 Landoni G, Lomivorotov VV, Nigro Neto C, et al; MYRIAD Study Group. Volatile anesthetics versus total intravenous anesthesia for cardiac surgery. N Engl J Med 2019;380(13):1214-1225

9 Bulow NM, Colpo E, Pereira RP, et al. Dexmedetomidine decreases the inflammatory response to myocardial surgery under mini-cardiopulmonary bypass. Braz J Med Biol Res 2016;49(4):e4646

10 Nagre AS, Jambures NP. Comparison of immediate extubation versus ultrafast tracking strategy in the management of off-pump coronary artery bypass surgery. Ann Card Anaesth 2018;21(2):129-133

11 Taware M, Sonkusale M, Deshpande R. Ultra-fast-tracking in cardiac anesthesia "Our Experience" in a rural setup. J Datta Meghe Inst Med Sci Univ 2017;12:110-114

12 Cook TM, Gibbison B. Analysis of 1000 consecutive uses of the ProSeal laryngeal mask airway by one anaesthetist at a district general hospital. Br J Anaesth 2007;99(3):436-439 